\title{
El enfoque por competencias y su relevancia en la actualidad: Consideraciones desde la orientación ocupacional en contextos educativos
}

\author{
An Approach by Competencies and Its Current Relevance: Considerations From \\ Occupational Guidance in Educational Context
}

\section{Abordagem por competências e sua relevância no presente: considerações a partir da Orientação Ocupacional em contextos educacionais}

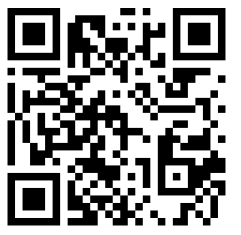

Jose Laurian Ramírez-Díaz Universidad Católica de Costa Rica

Cartago, Costa Rica

jramirezd@ucatolica.ac.cr

https://orcid.org/0000-0003-4644-9674

Recibido • Received • Recebido: 07 / 08 / 2018

Corregido • Revised • Revisado: 21 / 12 / 2019

Aceptado•Accepted • Aprovado: 31 / 03 / 2020

\begin{abstract}
Resumen: El enfoque por competencias se ha planteado como una de las propuestas contemporáneas para articular los procesos de formación con el mercado de trabajo. Sin una posición epistemológica específica, el concepto de competencias ha evolucionado en las últimas décadas hasta alcanzar una relevancia internacional destacada en la actualidad. Así mismo, con la consideración de constructos teóricos tales como la ocupación y empleabilidad, el enfoque por competencias ha ampliado su consideración hacia ámbitos variados y amplios entre los cuales se destacan los educativos, con planteamientos que le defienden y retractan. Este ensayo plantea una revisión teórica de los modelos más destacados del enfoque por competencias, así como de la relación que tienen con la orientación ocupacional y sus modelos de intervención, con el objetivo de que profesionales en la disciplina consideren sus aportes para el desarrollo de los procesos que realizan en distintos contextos, especialmente los educativos.
\end{abstract}

Palabras claves: Competencias; ocupación; orientación ocupacional; educación; empleabilidad. 
http://doi.org/10.15359/ree.24-2.23

ROR: https://ror.org/01t466c14 Universidad Nacional, Costa Rica

http://www.una.ac.cr/educare

educare@una.cr

\begin{abstract}
The approach by competencies explains the articulation between training processes and the work market. Without an obvious epistemic position, in the last decades, the concept of competencies has evolved toward an important framework to consider the work's human talent in many countries. Likewise, with consideration of the framework as occupation and employability, the approach by competencies has expanded its use to different fields and knowledge areas, such as educational ones among them, with proposals that defend it and retract it. This paper proposes a theoretical review of the most important models of approach by competencies, and the relation between these models with the occupational guidance and its intervention models so that the professionals in the area can considerer their contributions to developing the processes they implement in different contexts, especially in educational ones.
\end{abstract}

Keywords: Competencies; occupational guidance; counseling; education; employability.

Resumo. A abordagem por competências tem sido indicada como uma das propostas contemporâneas para articular processos de formação com o mercado de trabalho. Sem uma posição epistemológica específica, o conceito de competências evoluiu nas últimas décadas até alcançar uma relevante relevância internacional na atualidade. Da mesma forma, com a consideração de construtos teóricos como ocupação e empregabilidade, a abordagem baseada em competências ampliou sua consideração para áreas variadas e amplas, em meio a propostas que a defendem e a anulam. Este ensaio propõe uma revisão dos modelos mais destacados da abordagem baseada em competências, bem como a relação que eles têm com a Orientação Ocupacional e seus modelos de intervenção, com o objetivo de que os profissionais da disciplina considerem suas contribuições para o desenvolvimento dos processos realizados em diferentes contextos, especialmente os educacionais.

Palavras chaves: Competências; ocupação; orientação ocupacional; educação; empregabilidade.

\title{
Introducción
}

El concepto de competencias es común en la actualidad. Aunque tiene distintas acepciones, regularmente se consideran como una organización compleja de atributos que se basan en habilidades, conocimientos, actitudes y valores, los cuales se evalúan en situaciones determinadas (Costa Rica, Gobierno de la República, 2017), de modo que su incorporación a los procesos educativos se ha dado de forma cada vez más contundente como parte de las políticas actuales de preparación para el mundo del trabajo. El concepto de competencias en el contexto laboral fue planteado por David McClelland, en 1969, quien propuso la teoría de las necesidades y los tipos de motivación inspirado en las conductas, que sintetizó en: 1) el logro, traducido en el éxito y la búsqueda de sobresalir, 2) el poder, entendido como la influencia, el control y el reconocimiento de terceros y 3) la afiliación o pertenencia, que se interpreta a partir de las relaciones interpersonales cercanas y de amistad (McClelland, 2016).

El concepto de competencias surgió en la década de los 60; pero no fue sino hasta 2001 cuando, a partir del Proyecto Tuning en Europa, 175 universidades las incluyeron como parte de sus planes de estudio; actualmente se implementan en 19 países (Sánchez, 2011). En el

$$
2
$$

Jose Laurian Ramírez-Díaz

Los artículos de la Revista Electrónica Educare del Centro de Investigación y Docencia en Educación de la Universidad Nacional, Costa Rica, se comparten bajo términos de la Licencia Creative Commons: Reconocimiento, No Comercial, Sin Obra Derivada 3.0 Costa Rica. Las autorizaciones adicionales a las aquí delimitadas se pueden obtener en el correo: educare@una.cr 
contexto local, en 2004 dio inicio el Proyecto Tuning América Latina. Sánchez (2011) explica que unas 190 universidades han acogido el enfoque por competencias como criterio formativo, y su influencia se ha expandido a la educación secundaria, en especial dentro de la modalidad técnico profesional. Ejemplo de este avance ha sido la educación en alternancia, también llamada educación dual, la cual ha generado una serie de debates a nivel social y educativo en Costa Rica. Para comprender la relación del tema con la orientación ocupacional es necesario repasar algunos principios, por lo que se plantea un análisis en el contexto contemporáneo.

\section{Las competencias y el contexto actual}

El enfoque por competencias se ha consolidado especialmente por organismos internacionales. La Organización para la Cooperación y el Desarrollo Económico (OCDE) (2010) planteó, en su informe Habilidades y competencias del siglo XXI para los aprendices del nuevo milenio en los países de la OCDE, un marco de habilidades y competencias destacadas en las dimensiones de información, comunicación y ética, así como de impacto social. Debe recordarse que Costa Rica ha sido país invitado a incorporarse a este organismo, de modo que las competencias son, y serán, un considerando de suma relevancia. En el proceso de globalización, uno de los mayores responsables del surgimiento de este enfoque, las competencias son de suma relevancia. Cada vez, más ocupaciones tienden a robotizarse, en especial las operativas, y la información es un recurso más abundante gracias a las tecnologías modernas (OECD, 2017). Se requieren capacidades para discriminar aquella información que es útil de la que no, lo cual sustituye la memorización.

Pero no todo es acogida para el enfoque por competencias, ya que algunos entes detractores lo acusan de promover una visión pragmática, técnica, reduccionista, procedimental, de comportamientos fragmentados y discretos, con marcada influencia por el positivismo y con un posicionamiento producto de la globalización y las exigencias de las empresas, lo cual redujo la autenticidad humana al dominio de la realidad y no hacia su reflexión (Sánchez, 2011). Sin embargo, tal como se indicó, este enfoque es respaldado por organismos internacionales, de modo que su implementación se ha constituido en un requisito para la mejora de la competitividad. La negación de la realidad no la cambia en su fondo.

Por lo anterior, es necesario repasar algunas generalidades del enfoque por competencias en el ámbito educativo, de modo que profesionistas en orientación ocupacional destacados en estos ámbitos posean saberes básicos sobre ello, y se amplíe el criterio analítico sobre el debate actual, además de considerar la relación que guarda con la propuesta que, sobre educación de alternancia, se da a nivel nacional. Para lo anterior, se brinda un breve repaso sobre los modelos por competencias y sus características.

\section{Los modelos de gestión por competencias: Sus alcances y características}

Saracho (2005) explica que son tres los modelos básicos de la gestión por competencias, los 
cuales son ampliados y adaptados según la conveniencia y necesidades de las organizaciones. En este sentido, según este autor, se puede hablar del modelo de competencias distintivas, que considera las individualidades de cada persona como el criterio principal, el modelo de competencias genéricas en el cual destacan las pautas conductuales como elementos básicos para el logro de objetivo y las competencias funcionales, en las que se destaca el logro exitoso de los objetivos a partir de las consideraciones y demandas de los puestos en los que las personas gestionan sus acciones cotidianas.

Por su parte, Alles (2006, p. 26) explica que “la preocupación por cómo mejorar en materia de comportamientos o conductas humanas viene de muy antiguo", de modo que aunque es el concepto ha sido tratado desde hace algunas décadas, las competencias se derivan del conocimiento y de ciertas características de la personalidad, una situación que ha sido tratada en distintos momentos históricos con base en criterios socioculturales. Así mismo, la motivación mejora a partir de la identificación de características y capacidades personales, la consideración de las necesidades de la organización y de la implementación de un sistema adecuado de evaluación del desempeño, basado en el principio dual de premio y castigo, elementos básicos para este enfoque (Alles, 2006). El paradigma tradicional educativo muestra una postura coercitiva del error, pero los modelos por competencias le conciben, con límites racionales, como oportunidades de mejora.

Considerando lo anterior, al referir las competencias desde criterios que se plantean como elementos observables, por ejemplo, las conductas. La descripción que hacen los modelos mencionados dan énfasis a la evaluación de los comportamientos como el principio para entrenar, desarrollar y validar las competencias; se trasciende el planteamiento educativo tradicionalista que privilegiaba la habilidad memorística, de modo que afronta a las personas a aplicar el conocimiento en distintas situaciones; valida el aprendizaje como un proceso escalonado e integral en la que los errores forman parte; da enfasis a procesos más integrales en los que para la adquisición y asimilación de saberes se integran al saber conocer, el saber hacer, saber ser y el saber convivir, algo de que se hablará más adelante.

Ahora bien, es importante recordar que, entre los principios del conductismo, los comportamientos se modifican con base en estímulos de reforzamiento, ya sea para mantener las conductas o prevenirlas (Feist, Feist y Roberts, 2014), y la motivación es un elemento presente en el entrenamiento y desarrollo de las competencias. De esta forma, es importante considerar que como parte del cambio de paradigma planteado en los modelos de competencias con respecto a la educación tradicional, la motivación no se alcanza a partir del éxito, sino se considera como una dimensión humana basada en el deseo de aprender. Explica Oppenheimer (2014) que una de las situaciones que más le llamó la atención en una de sus visitas a Silicon Valley, en las que entrevistó a directores de empresas de innovación, fue que los antecedentes que más destacaron como parte de sus mayores éxitos no fueron logros, sino errores que les 
enseñaron a hacer las cosas de formas distintas.

Si bien el asunto de analizar si los errores pueden ser oportunidades para incentivar, en las personas, la motivación por aprender no forma parte de los objetivos en este texto, particularmente se considera que estos pueden ser situaciones sumamente oportunas para la mejora en los entornos de estudio y de trabajo. Si se les canalizan de forma adecuada, algo que para los modelos de competencias es sumamente válido y oportuno, permiten a las personas ensayar comportamientos adecuados ante experiencias distintas, preveniendo las respuestas comportamentales que carecieron de eficacia ante situaciones específicas y replicando aquellas que derivaron en los resultados más adecuados; algo que puede ser sumamente valioso en el cumplimiento de las metas en entornos formativos y laborales, lo cual no es indistinto al principio de reforzamiento que proponen las teorías conductistas.

De esta forma, las competencias consideran los comportamientos en situaciones

Tabla 1: Modelos de gestión por competencias

\begin{tabular}{|c|c|c|c|c|}
\hline Modelo & Características & & Niveles & Conceptos base \\
\hline 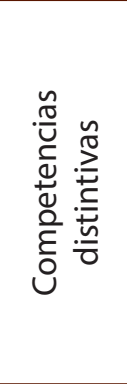 & $\begin{array}{l}\text { Se centra en las características } \\
\text { que diferencian a cada } \\
\text { persona, para crear un perfil } \\
\text { individual a partir de los } \\
\text { resultados de su trabajo, } \\
\text { mediante la generalizaciónd e } \\
\text { competencias }\end{array}$ & $\begin{array}{l}\text { 1) } \\
\text { 2) } \\
3\end{array}$ & $\begin{array}{l}\text { Estáticas: aprovechamiento de } \\
\text { recursos tangibles } \\
\text { Coordinación: funcionales o de } \\
\text { cohesión organizacional directiva } \\
\text { Dinámicas: para la adaptación, } \\
\text { integración, construcción o } \\
\text { reconfiguración de nuevas } \\
\text { competencias y adaptación al } \\
\text { cambio }\end{array}$ & Talento humano \\
\hline 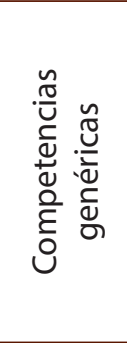 & $\begin{array}{l}\text { Se fija en las conductas } \\
\text { y el logro de resultados } \\
\text { desde la consecución de } \\
\text { objetivos o metas, mediante } \\
\text { el desempeño según las } \\
\text { ocupaciones y las similitudes } \\
\text { entre estas }\end{array}$ & $\begin{array}{l}\text { 1) } \\
\text { 2) } \\
\text { 3) }\end{array}$ & $\begin{array}{l}\text { Instrumentales: comprender } \\
\text { y manipular ideas, métodos y } \\
\text { destrezas varias } \\
\text { Interpersonales: expresión de } \\
\text { sentimientos, criticidad y autocrítica } \\
\text { Sistémicas: comprensión, } \\
\text { sensibilidad y conocimiento } \\
\text { sistémicas de la organización }\end{array}$ & $\begin{array}{l}\text { Mejores prácticas y } \\
\text { Benchmarketing }\end{array}$ \\
\hline 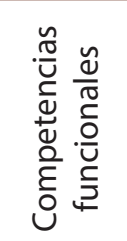 & $\begin{array}{l}\text { Se interesa en el desempeño } \\
\text { para gestionar el logro de } \\
\text { objetivos específicos de la } \\
\text { organización, así como el logro } \\
\text { de resultados mínimos }\end{array}$ & $\begin{array}{l}\text { 1) } \\
\text { 2) }\end{array}$ & $\begin{array}{l}\text { Cardinal: demandas generales } \\
\text { adheridas a la visión organizacional } \\
\text { Específica: aplicada a grupos } \\
\text { definidos }\end{array}$ & $\begin{array}{l}\text { Competencias técnicas, } \\
\text { normalización de } \\
\text { competencias y } \\
\text { certificación por } \\
\text { competencias }\end{array}$ \\
\hline
\end{tabular}

Nota: Elaboración propia con base en Saracho (2005) y Alles (2017). 
particulares, los cuales son, en principio, elementos observables que manifiestan rasgos de la personalidad, por lo que constituyen la unidad observable de las competencias (ver Tabla 1).

Los modelos por competencias distintivas y competencias genéricas, propuestos por David McClelland y William Byham, respectivamente, son considerados conductistas; el de competencias funcionales, creado y desarrollado por Sydney Fine, se inclina por un análisis ocupacional de naturaleza constructivista (Saracho, 2005).

En el ámbito costarricense, el Ministerio de Educación Pública (en adelante MEP), plantea políticas transversales que integran las siguientes competencias:

- Cultura ambiental para el desarrollo sostenible

- Educación integral de la sexualidad

- Educación para la salud

- Vivencia de los derechos humanos para la democracia y la paz (Méndez, 2007).

El Centro Latinoamericano de Administración para el Desarrollo (CLAD) (2016) ha

Tabla 2: Pilares del saber

\begin{tabular}{ll}
\hline \multicolumn{1}{c}{ Pilar } & \multicolumn{1}{c}{ Descripción } \\
\hline Saber ser & $\begin{array}{l}\text { Pensamiento crítico y autónomo de construcción del juicio propio, manifiesta los } \\
\text { conocimientos adquiridos a lo largo de la vida }\end{array}$ \\
Saber conocer & $\begin{array}{l}\text { La adquisicion de conocimientos mediante la atención, la memoria y el pensamiento: un } \\
\text { medio y un fin de la vida humana }\end{array}$ \\
Saber hacer & Relacionado al ejercicio profesional y a las competencias propiamente \\
Saber convivir & $\begin{array}{l}\text { Vinculado a las relaciones interpersonales e inteligencia emocional, así como a los } \\
\text { comportamientos adquiridos a partir de la experiencia }\end{array}$ \\
\hline
\end{tabular}

Nota: Elaboración propia con base en Delors (1996) y CLAD (2016).

propuesto, en concordancia con lo planteado por Delors (1996) y la Organización de las Naciones Unidas para la Educación, la Ciencia y la Cultura (UNESCO), la consideración de competencias 
con base en los pilares del saber (Tabla 2).

Así, en atención al criterio formativo de la orientación ocupacional, se plantea el análisis de las competencias desde el contexto de los aprendizajes y su evaluación.

\section{El aprendizaje y evaluación de las competencias}

Las competencias se basan en principios de aprendizaje, que implican cambios en el comportamiento (Carlson y Birkett, 2017), por lo que su entrenamiento y evaluación deben conocerse por anticipado (A. A. Calvo, comunicación personal, 23 de marzo, 2018). Las competencias distinguen ciertos elementos que pueden referirse "como el conjunto de conocimientos, actitudes y destrezas necesarias para desempeñar una ocupación dada" (Venegas, 2011, p. 10). En este sentido, existe un criterio común entre las competencias y la orientación ocupacional, el cual deriva en uno de los focos de atención aquí planteados: la ocupación. Muchas veces se ha defendido el principio cualitativo característico de la mayoría de procesos de orientación ocupacional, pero este criterio es insuficiente para lo aquí tratado, ya que la evaluación por competencias es más compleja. Existen rasgos colectivos (líneas base) que no discuten con la integridad individual y que, como parámetro comparativo, coadyuvan a mejorar procesos educativos y laborales, los cuales están vinculados a la capacidad de respuesta que tienen las personas para afrontar y resolver las vivencias cotidianas (Bunge, 2014), lo cual

Tabla 3: Síntesis instrumental, según modelo evaluativo de Kikrpatrick

\begin{tabular}{lll}
\hline \multicolumn{1}{c}{ Nivel } & \multicolumn{1}{c}{ Criterios de evaluación } \\
\hline I. Reacción o & Responde a la pregunta: ¿Le gustó la actividad a las personas participantes? \\
II. Aprendizaje & $\begin{array}{l}\text { Busca determinar en qué medida los sujetos participantes valoraron la capacitación. } \\
\text { Busca responder o da respuesta a la pregunta: ¿Las personas participantes desarrollaron } \\
\text { los objetivos en la acción de capacitación? } \\
\text { Busca determinar el grado en que los sujetos participantes han logrado los objetivos de } \\
\text { aprendizaje establecidos para la capacitación. }\end{array}$ \\
III. Aplicación o & $\begin{array}{l}\text { Da respuesta a la pregunta: ¿Están las personas participantes utilizando en su trabajo las } \\
\text { transferencia }\end{array}$ & $\begin{array}{l}\text { competencias desarrolladas? } \\
\text { Pretende determinar si los sujetos participantes han transferido las habilidades y el } \\
\text { conocimiento adquirido de la capacitación a sus funciones, identificando aquellas variables } \\
\text { que pudiesen haber afectado el resultado. }\end{array}$ \\
& $\begin{array}{l}\text { Responde a la pregunta: ¿Cuál es el impacto operacional? } \\
\text { IV. Resultadosopósito es determinar el impacto en los resultados que ha producido una acción de } \\
\text { capacitación. }\end{array}$ \\
\hline
\end{tabular}

Nota: A partir de Cabrera (2005). 
http://doi.org/10.15359/ree.24-2.23

ROR: https://ror.org/01t466c14 Universidad Nacional, Costa Rica

http://www.una.ac.cr/educare

educare@una.cr

constituye un pilar de la gestión por competencias. Además, particularmente se considera que son dos los ámbitos de evaluación de las competencias: uno cualitativo, que analiza criterios con base en el proceso de aprendizaje y otro cuantitativo, que tiende a evaluar el impacto de la competencia. Al respecto, Kirkpatrick (1975, citado por Cabrera, 2005) distingue cuatro niveles de evaluación (Tabla 3).

Considerando los niveles evaluativos anteriores, es posible que los procesos sean más sencillos de evaluar de forma casi que inmediata, lo cual deja de lado el impacto que tienen entre las personas beneficiarias. Sin embargo, particularmente se considera que es necesario dar un seguimiento en el corto y mediano plazo para evaluar, de forma adecuada, no solo los resultados inmediatos que se pueden observar, sino aquellos que derivan en cambios más profundos, algo que el modelo anterior promueve y que con un enfoque basado en competencias puede ser favorecido, ya que se pueden identificar comportamientos que, evaluados transversalmente en el tiempo, pueden conformarse en criterios objetivos que sean valorados para el propósito aquí analizado.

Para ofrecer un ejemplo de lo anterior, si como objetivo de un proceso formativo se tuviera la sensibilización y promoción del respeto por la diversidad entre las personas, una de las temáticas de mayor interés en la actualidad, la evaluación de cuánto comprendieron sobre los conceptos básicos no sería suficiente, ya que esto no garantizaría que ello se tradujera en acciones específicas de mejora; en cambio, si se evalúan los comportamientos que las personas presentan en un plazo de tiempo determinado, esto daría oportunidad para valorar el impacto que ha tenido dicho proceso en las personas y en sus estilos de vida, tanto en el saber hacer (aplica los conocimientos en sus acciones), en el saber ser (se apropia del respeto por la diversidad como uno de sus valores) y del saber convivir (practica el respeto hacia sí mismas y a las demás personas), lo cual se constituiría en la medición del impacto que se obtuvo.

De esta forma, la evaluación de los procesos de orientación ocupacional que involucren competencias requieren integrar criterios de evaluación del proceso y de su impacto, con el propósito de mejorar el desempeño en el ámbito ocupacional (Gertler et ál., 2011). Esto es de necesaria consideración porque las competencias se dividen en grupos, definidos por el CLAD (2016) en: 1) Transversales, que son demandadas a todas las personas en la organización, 2) Directivas, descritas para jefaturas o para quienes tienen personal a cargo y 3) Técnico o profesionales, para ocupaciones con funciones específicas o especializadas. Así mismo, se consideran, para su evaluación, niveles de logro o dominio, donde una referencia adecuada la ofrece la taxonomía SOLO, la cual se define a partir del planteamiento de niveles de dominio de las competencias con base en los comportamientos desarrollados como criterios que demuestran que las personas las han incorporado. En la Tabla 4 se describe esta taxonomía.

Con base en la taxonomía SOLO, en el ámbito costarricense, los niveles tienden derivarse en: 1) Deficiente (preestructural), 2) Regular (uniestructural), 3) Bueno (multiestructural), 4) Muy bueno (relacional) y 5) Excelente (abstracto). Respecto a estas escalas de evaluación, A. A. Calvo (Comunicación personal, 23 de marzo, 2018) explica que en las competencias se consideran dos

8

Jose Laurian Ramírez-Díaz

Los artículos de la Revista Electrónica Educare del Centro de Investigación y Docencia en Educación de la Universidad Nacional, Costa Rica, se comparten bajo términos de la Licencia Creative Commons: Reconocimiento, № Comercial, Sin Obra Derivada 3.0 Costa Rica. Las autorizaciones adicionales a las aquí delimitadas se pueden obtener en el correo: educare@una.cr 
Tabla 4: Descripción de la taxonomía SOLO

\begin{tabular}{|c|c|c|c|c|}
\hline \multicolumn{5}{|c|}{ Nivel } \\
\hline $\begin{array}{c}\text { Preestructural } \\
1\end{array}$ & $\begin{array}{c}\text { Uniestructural } \\
2\end{array}$ & $\begin{array}{c}\text { Multiestructural } \\
3\end{array}$ & $\begin{array}{c}\text { Relacional } \\
4\end{array}$ & $\begin{array}{l}\text { Abstracto } \\
5\end{array}$ \\
\hline $\begin{array}{l}\text { No existe } \\
\text { evidencia o } \\
\text { comprensión de } \\
\text { la competencia }\end{array}$ & $\begin{array}{l}\text { La persona identifica } \\
\text { comportamientos } \\
\text { básicos en } \\
\text { procedimientos } \\
\text { sencillos }\end{array}$ & $\begin{array}{l}\text { La persona se } \\
\text { enfoca en aspectos } \\
\text { relevantes de } \\
\text { comportamientos } \\
\text { en forma aislada }\end{array}$ & $\begin{array}{l}\text { La persona } \\
\text { integra aspectos } \\
\text { relevantes de } \\
\text { comportamientos } \\
\text { en forma coherente }\end{array}$ & $\begin{array}{l}\text { La persona } \\
\text { generaliza aspectos } \\
\text { relevantes de } \\
\text { comportamientos en } \\
\text { distintas funciones }\end{array}$ \\
\hline
\end{tabular}

Nota: A partir de Biggs y Collis (1982).

criterios: la amplitud, relacionada con el qué evaluar, y la profundidad, vinculada al cómo. Valverde et ál. (2012) explican que las actividades formativas deben ofrecer "la oportunidad de conocer cuáles son sus resultados de aprendizaje en lo que se refiere al 'qué' se ha aprendido y al 'cómo' habría podido hacerse" (p. 52). Las competencias se consideran desde criterios comportamentales de modo que, en contextos educativos, la profundidad podría considerarse desde situaciones hipotéticas y, por tanto, evaluada por respuestas comportamentales y sus resultados.

La evaluación "necesita ser considerada como un proceso de recogida de evidencias (a través de actividades de aprendizaje) y de formulación de valoraciones sobre la medida y la naturaleza del progreso del estudiante, según unos resultados de aprendizaje esperados" (Valverde et ál., 2012, p. 53), por lo que las competencias deben homologarse según las demandas organizacionales o del contexto, y los instrumentos de evaluación pueden considerar entrevistas y cuestionarios de valoración del aporte individual al mejoramiento de los procesos (Albino, 2018). Uno de los objetivos fundamentales de la implementación de competencias es disminuir brechas, por lo que la mejora continua es rectora para estos modelos de gestión, para lo cual es necesario una metodología adecuada de evaluación (Smedick y Rice, 2018).

Hasta aquí se consideraron las competencias en su aprendizaje y evaluación. Ahora se hará referencia a la relación de esto con la orientación ocupacional.

\section{Las competencias y la orientación ocupacional}

Existen distintas concepciones de la disciplina de orientación que responden a sus ámbitos de acción. Respecto a la orientación ocupacional, la Organización Internacional del Trabajo explica:

Es un aspecto importante del trabajo que introduciría el concepto de rol. La ocupación haría referencia a la actividad que realiza una persona para obtener una fuente de ingresos 
http://doi.org/10.15359/ree.24-2.23

ROR: https://ror.org/01t466c14 Universidad Nacional, Costa Rica

http://www.una.ac.cr/educare

educare@una.cr

y que determina su posición social, su rol social identificable y con significado tanto para el ocupante de ese rol como para las demás personas que interactúan con él. (Hall, 1986 citado por Mosca y Santiviago, 2017, p. 14)

La ocupación se trata de un considerando de distintos ámbitos particulares, colectivos y sociales, los cuales configuran y construyen la experiencia de cada persona desde constructos como el trabajo y su influencia en el proyecto, la calidad y el estilo de vida a nivel individual y colectivo. Actualmente se considera el término de ocupación en sustitución al de puestos, lo que desplaza, a su vez, al organigrama institucional (A. A. Calvo, comunicación personal, 23 de marzo, 2018).

La ocupación se comprende desde la cotidianeidad, lo que "incluye el estudio, lo laboral, el tiempo libre, lo familiar, lo social, etc. Siempre pensando desde la acción concreta, aquellas actividades que hacemos todos los días, en definitiva, cómo y en qué ocupamos nuestro tiempo de vida" (Mosca y Santiviago, 2017, p. 14). También se relaciona con la promoción de la salud, donde figuran los considerandos del envejecimiento activo y la terapia ocupacional, que establecen una relación entre ocupación, salud y bienestar (Aguilar-Parra et ál., 2016). En otras palabras, las actividades de ocio y las responsabilidades particulares y colectivas en ámbitos ocupacionales conciben a las personas integralmente, desde sus roles vitales.

Es frecuente emplear test vocacionales para valorar interes, habilidades y aptitudes en

Tabla 5: Modelos de intervención de la orientación ocupacional

\begin{tabular}{|c|c|}
\hline Modelo & Características \\
\hline Consejo o counseling & $\begin{array}{l}\text { Se basa en un enfoque preventivo personalizado de entrevistas y técnicas de consejo, } \\
\text { cuyo objetivo es el desarrollo de la aceptación, autoconocimiento, los recursos personales } \\
\text { y el crecimiento emocional, se centra en la persona y no en sus problemas. Está basado } \\
\text { en una relación entre la persona profesional en orientación y la persona orientada. }\end{array}$ \\
\hline Programas & $\begin{array}{l}\text { Considera actividades planificadas, promueve el trabajo interdisciplinario, cuyo objetivo } \\
\text { es satisfacer necesidades de un contexto o grupos de personas, enriquecer, desarrollar o } \\
\text { entrenar competencias específicas. }\end{array}$ \\
\hline Consulta & $\begin{array}{l}\text { Este modelo se basa en una relación entre dos profesionales, lo cual la diferencia } \\
\text { del modelo de counseling, ya que en este se atienden las demandas de la persona } \\
\text { consultante. En los ámbitos educativos tiene como objetivo la consultoría al cuerpo } \\
\text { docente y a las familias, así como a la institución educativa. }\end{array}$ \\
\hline
\end{tabular}

Nota: Con base en Orozco (2016). 
los procesos de orientación (Fuentes, 2010), lo que comulga con varios planteamientos de la gestión por competencias, con criterios de líneas base. Así mismo, la mayoría de carreras a nivel técnico, parauniversitario y nivel terciario presentan perfiles de entrada y de salida, los cuales son insumos de consulta que demuestran, en opinión particular, que criterios como los conocimientos, las actitudes y destrezas son un referente útil para utilizar en procesos de orientación ocupacional, pues son considerados como rasgos competentes. Es importante considerar, aquí, los modelos de intervención con los que cuenta la disciplina (Tabla 5).

Las competencias y la orientación poseen criterios comúnes referidos a ámbitos ocupacionales que integran dimensiones y tareas del desarrollo, no solo en los contextos de trabajo, sino en roles vitales. Existen teorías y enfoques que plantean la integración de la cotidianeidad dentro de los procesos de orientación ocupacional, como puede ser la teoría del desarrollo humano y la incorporación de las dimensiones cognitiva y social (Papalia y Martorell, 2017), lo que demuestra que la gestión por competencias puede constituirse desde el principio de integridad del ser humano, un pilar para esta disciplina. Es relevante que en los procesos de orientación ocupacional se consideren las competencias como un elemento sustantivo, primero para responder a las demandas y tendencias contemporáneas $y$, segundo, porque plantean una estrategia adecuada para los procesos modernos de búsqueda de trabajo. Además, es importante que cada profesionista prevenga la deshumanización en el empleo de este enfoque, situación que puede darse en cualquier otra intervención. Las competencias no deben considerarse solo en ámbitos de trabajo, sino en varios contextos como el educativo, individual e interpersonal, de modo que su empleo contribuya en ampliar los contextos inverventivos de la orientación ocupacional y sus beneficios subsecuentes.

El cambio de paradigma generacional actual, en el que el "compromiso hacia las causas no es tratado en ámbitos de arraigo por tradiciones, sino a ideales y esquemas mentales de conveniencia" (Ramírez, 2017, p. 46), plantea una disposición creciente de las personas a hacer cambios sustanciales en su vida, si consideran que su realización personal será favorecida, de modo que no permanecen adheridas a esquemas, como sucedía anteriormente, sino que varían según los intereses y la etapa del desarrollo. Longo (2004) expone que el contrato psicológico anteriormente radicaba en la percepción de seguridad que una persona percibía al tener un trabajo, pero que en la actualidad ha cambiado hacia la empleablidad. Esta se basa en la realización personal mediante el impacto de las acciones particulares en distintas áreas, donde deben considerarse evaluaciones cualitativas (aprendizajes del proceso) y cuantitativas (comportamientos); en sistemas de motivación, actitudes, conocimientos, habilidades, valores, aptitudes y capacidades laborales necesarias para un desempeño satisfactorio de alguna ocupación, según plantean Arias et ál. (2007).

Las nuevas ocupaciones ofrecen y demandan competencias. No es casualidad que, entre las empresas más exitosas en la actualidad existan varias que dedican sus actividades para satisfacer 
necesidades mediante métodos y formas innovadoras, una evidencia de que la ocupación, las competencias y la empleabilidad caminan de la mano. La orientación ocupacional debe incluir las competencias en sus procesos. Así, por ejemplo, en un proceso de intervención desde el modelo de counseling, el favorecimiento del autoconocimiento, los recursos individuales, la autoaceptación y el crecimiento emocional derivan en el entrenamiento de competencias a nivel individual, que promueven la distinción de la persona en su entorno interpersonal. Desde el modelo por programas se pueden planificar acciones para satisfacer ciertas necesidades de un colectivo. Sean cualesquiera los contextos, se entrenan competencias que requieran de una intervención determinada para mejorar ciertos procesos o tareas en entornos y tareas específicos, se establecen líneas determinadas para mejorar prácticas, se generan modelos competentes de comunicación y de trabajo en equipo, por ejemplo. Finalmente, mediante la consultoría, se pueden favorecer procesos de mejora continua, una estrategia muy usada para implementar modelos de gestión por competencias particulares o específicas en un colectivo en aprovechamiento de competencias técnicas que, por su constitución, cada ocupación demanda o los perfiles formativos favorecen.

\section{Conclusiones}

El entrenamiento por competencias en ámbitos educativos es una realidad actual, se posiciona cada vez más en las políticas educativas y demanda una intervención integral. La preparación para el mundo del trabajo no solo pretende enseñar a las personas a cumplir con funciones específicas, sino que desde los enfoques de orientación ocupacional trata de que las personas alcancen la plenitud a partir de sus actividades cotidianamente productivas; ajeno a ello, se corre el riesgo de deshumanizar procesos cuyo objetivo es brindar herramientas para la autorrealización de las personas beneficiarias mediante desempeños adecuados en actividades ocupacionales. Para esto, la motivación debe considerarse como una relevante constante, ya que los cambios pueden beneficiarse, si se establecen a partir de ella, lo cual favorece comportamientos competentes ante situaciones específicas.

La perspectiva que plantea la orientación ocupacional coadyuva a la intervención integral de las dimensiones que conforman a cada ser humano, por lo que puede ser de gran valía y beneficio incorporar las competencias en sus procesos, no solo desde los ámbitos de trabajo, sino desde la integridad que se concibe en la dimensión ocupacional, la cual involucra distintas esferas del ser humano, incluidas la motivación, la empleabilidad, así como los procesos de desarrollo humano y relaciones internacionales, entre otras.

La evaluación de estos procesos debe incorporar estrategias más amplias y variadas, tanto cualitativas como cuantitativas, lo que demanda actualizar los métodos respectivos utilizados por profesionales en orientación ocupacional, sin dejar de lado el trabajo interdisciplinario, sumamente valioso y necesario en la actualidad. 


\section{Referencias}

Aguilar-Parra, J. M., López-Liria, R., Fernández-Batanero, J. M., Padilla-Góngora, D. y Álvarez, J. (2016). Influencia de la ocupación en actividades de la vida diaria y de ocio sobre la salud real de la persona mayor. Psychology, Society \& Education, 8(2), 135-147. https://doi. org/10.25115/psye.v8i2.554

Albino, G. (2018). Technical and behavioral competencies on performance evaluation: Petrek leaders' perspectives. SAGE Open, 8(2), 1-12. https://doi.org/10.1177/2158244018780972

Alles, M. (2006). Desarrollo del talento humano basado en competencias. Granica. https://www. academia.edu/33494469/Desarrollo del Talento Humano Basado en Competencias de Alles Martha

Alles, M. (2017). Selección por competencias (2ª ed.). Granica.

Arias, L., Portilla, L. M. y Florez, M. E. (2007). Competencias y empleabilidad. Scientia Et Technica, 1(37), 379-382. https://revistas.utp.edu.co/index.php/revistaciencia/article/view/4125

Biggs, J. B. y Collis, K. F. (1982). Evaluating the quality of learning: The SOLO taxonomy (Structure of th obsrved leraning outcome). Academic Press.

Bunge, M. (2014). La ciencia, su método y su filosofía. Editorial Sudamericana.

Cabrera, J. A. (2005). Evaluación de la capacitación y de su impacto en el desempeño individual y organizacional. $V$ Congreso Internacional Virtual de Educación, 1-46. http://sedici.unlp. edu.ar/bitstream/handle/10915/24726/Documento completo.pdf?sequence=1

Carlson, N. R. y Birkett, M. A. (2017). Physiology of behavior (12 ${ }^{\mathrm{a}}$ ed.). Pearson.

Centro Latinoamericano de Administración para el Desarrollo (CLAD). (2016). Guía referencial iberoamericana de competencias laborales en el sector público. Bogotá, Colombia: Autor. https://cladista.clad.org/handle/123456789/8193

Costa Rica, Gobierno de la República. (2017). Marco nacional de cualificaciones de la educación y la formación técnica profesional de Costa Rica. Autor.

Delors, J. (Preside). (1996). Los cuatro pilares de la educación (Cap. 4). En J. Delors (preside), La educación encierra un tesoro (pp. 95-109). Santillana. Ediciones UNESCO. innovacioneducativa.uaem.mx:8080/innovacioneducativa/web/.../educacion tesoro.p...

Feist, J., Feist, G. J. y Roberts, T-A. (2014). Teorías de la personalidad (8ª ed.). McGraw-Hill.

Fuentes, M.T. (2010). La orientación profesional para elegir fundamentadamente una ocupación: 
Propuesta alternativa. Revista Mexicana de Psicología, 27(2), 237-246. http://www.redalyc. org/articulo.oa?id=243016324012

Gertler, P. J., Martínez, S., Premand, P., Rawlings, L. B. y Vermeersch, C. M. J. (2011). La evaluación de impacto en la práctica. Washington DC: BID y Banco Mundial. https://doi.org/10.1596/978$\underline{0-8213-8681-1}$

Longo, F. (2004). Mérito y flexibilidad. La gestión de las personas en las organizaciones del sector público. Paidós.

McClelland, D. C. (2016). The achieving society. Pickle Partners Publishing.

Méndez, J. (2007). Diseño curricular bajo el modelo de educación basada en normas por competencia. MEP. https://www.mep.go.cr/sites/default/files/programadeestudio/ programas/contabilidad-11.pdf

Mosca, A. y Santiviago, C. (2017). Conceptos y herramientas para aportar a la orientación vocacional ocupacional de los jóvenes. Universidad de la República. http://www2. compromisoeducativo.edu.uy/sitio/wp-content/uploads/2013/10/MANUALCONCEPTOS-Y-HERRAMIENTAS-OVO.pdf

Oppenheimer, A. (2014). ¡Crear o morir! la esperanza de Latinoamerica y las cinco claves de la innovación. Vintage Español.

Organisation for Economic Co-operacition and Development (OECD). (2017). OECD Skills Outlook 2017: Skills and global value chains. OECD Publishing. https://doi. org/10.1787/9789264273351-en

Organización para la Cooperación y el Desarrollo Económicos. (2010). Habilidades y competencias del siglo XXI para los aprendices del nuevo milenio en los países de la OCDE. ITE. http:// observatoriocultural.udgvirtual.udg.mx/repositorio/handle/123456789/181

Orozco, A. M. (2016). Fundamentos sobre la orientación profesional actual en la construcción de proyectos académico-profesionales. Revista Mexicana de Orientación Educativa, 13(31), 37-44. http://remo.ws/remo-31/

Papalia, D. E. y Martorell, G. (2017). Desarrollo humano (13a ed.). McGraw-Hill.

Ramírez, J. L. (2017). Habilidades profesionales y su impacto en la gestión de empleo y el mercado laboral: Una arista desde el talento y la innovación de la generación millenials. En P. Astudillo, D. Cisneros y K. Luna (Comps.), Memorias científicas del $2^{\circ}$ Congreso Internacional de Administración de empresas (pp. 43-61). CIDE Editorial. https://cidecuador. 


\section{org/.../congresos/2017/...congreso-internacional-de-administracion-...}

Sánchez, J. (2011). Hacia un currículo transdisciplinario: Una mirada desde el pensamiento complejo. Revista de Teoría y Didáctica de las Ciencias Sociales, 17, 143-164. http://www. redalyc.org/articulo.oa?id $=65221619010$

Saracho, J. M. (2005 ). Un modelo general de gestión por competencias. RIL Editores.

Smedick, B. y Rice, E. (2018). An overview of leadership competenciesand assessment considerations. En K. K. Smith, G. S. Rooney y G. Spencer (Eds.), Leadership Development for Career Readiness in University Settings (pp. 55-70). Wiley Periodicals. https://doi. org/10.1002/yd.20279

Valverde, J., Revuelta, F. I. y Fernández, M. R. (2012). Modelos de evaluación por competencias a través de un sistema de gestión de aprendizaje. Experiencias en la formación inicial del profesorado. Revista Iberoamericana de Educación, 60, 51-62. https://rieoei.org/RIE/issue/ view/37

Venegas, P. (2011). Gestión de la educación basada en competencias: Elementos para su interpretación en el contexto de la administración de la educación. Revista Gestión de la Educación, 1(1), 1-27. https://doi.org/10.15517/rge.v1i1.8611 